\title{
Clinical diagnosis of ventilator associated pneumonia revisited: comparative validation using immediate post-mortem lung biopsies
}

Neus Fàbregas, Santiago Ewig, Antoni Torres, Mustafa El-Ebiary, Josep Ramirez, Jorge Puig de la Bellacasa, Torsten Bauer, Hernan Cabello

\begin{abstract}
Background-A study was undertaken to assess the diagnostic value of different clinical criteria and the impact of microbiological testing on the accuracy of clinical diagnosis of suspected ventilator associated pneumonia (VAP).

Methods-Twenty five deceased mechanically ventilated patients were studied prospectively. Immediately after death, multiple bilateral lung biopsy specimens (16 specimens/patient) were obtained for histological examination and quantitative lung cultures. The presence of both histological pneumonia and positive lung cultures was used as a reference test.

Results-The presence of infiltrates on the chest radiograph and two of three clinical criteria (leucocytosis, purulent secretions, fever) had a sensitivity of $69 \%$ and a specificity of $75 \%$; the corresponding numbers for the clinical pulmonary infection score (CPIS) were $77 \%$ and $42 \%$. Non-invasive as well as invasive sampling techniques had comparable values. The combination of all techniques achieved a sensitivity of $85 \%$ and a specificity of $50 \%$, and these values remained virtually unchanged despite the presence of previous treatment with antibiotics. When microbiological results were added to clinical criteria, adequate diagnoses originating from microbiological results which might have corrected false positive and false negative clinical judgements $(n=5)$ were countered by a similar proportion of inadequate diagnoses $(n=6)$.

Conclusions-Clinical criteria had reasonable diagnostic values. CPIS was not superior to conventional clinical criteria. Non-invasive and invasive sampling techniques had diagnostic values comparable to clinical criteria. An algorithm guiding antibiotic treatment exclusively by microbiological results does not increase the overall diagnostic accuracy and carries the risk of undertreatment.

(Thorax 1999;54:867-873)
\end{abstract}

Keywords: ventilator associated pneumonia; clinical diagnosis; sampling; lung biopsies
Clinical criteria have repeatedly been considered as inappropriate for the diagnosis of ventilator associated pneumonia (VAP). Necropsy studies conducted in the early 1980s suggested that the use of clinical parameters to diagnose VAP leads to overdiagnosis ${ }^{1}$ or, even worse, underdiagnosis, particularly in patients with adult respiratory distress syndrome (ARDS). ${ }^{2}$ More recently, additional evidence for this view has been put forward from evaluation of clinical criteria by microbiological ${ }^{3}$ or post-mortem histological reference tests. ${ }^{4-6}$ As a consequence, some authors have argued that the diagnosis of VAP should be guided by the quantitative culture results of bronchoscopically retrieved lower respiratory tract samples. ${ }^{7}$ A corresponding algorithm would imply that patients with a clinical suspicion of pneumonia but negative microbiological results would not receive antibiotics. Clinical criteria for the suspicion of VAP continue to form the starting point of diagnostic evaluation. It therefore remains important to determine the accuracy of these criteria.

A major methodological problem in this regard is the accuracy of the reference test. During the last five years several immediate post-mortem studies have validated diagnostic techniques using histological or microbiological examination of lung tissue samples. ${ }^{468-10}$ These studies have established considerable limitations in the diagnostic accuracy of quantitative cultures of lower respiratory tract samples and have raised concerns about using these techniques as a reference for clinical criteria. Clinical parameters have been validated in a previous report from our group in which the histological examination of a single lung biopsy sample was used as the reference test. ${ }^{4}$ However, as other workers have shown, ${ }^{8}$ a single lung biopsy sample may frequently underdiagnose pneumonia. Moreover, histological evidence of pneumonia may reflect any stage during its evolution but not necessarily the active inflammation responsible for clinical symptoms.

We therefore chose to validate clinical criteria by both the presence of histological pneumonia and positive quantitative lung tissue cultures as reference tests most probably reflecting clinically symptomatic pneumonia. 
We included clinical criteria as defined by Johanson et $a l^{11}$ as well as the clinical pulmonary infection score (CPIS) as proposed by Pugin et al. ${ }^{12}$ To determine the impact of microbiological criteria on the accuracy of the diagnosis of pneumonia we compared the performance of clinical criteria and different sampling techniques and also analysed the diagnostic results when adding the microbiological results to the clinical diagnoses. This approach allows an estimate of the potential diagnostic contribution of sampling techniques in everyday practice when evaluating patients with clinically suspicious VAP.

\section{Methods}

PATIENTS

Twenty five patients whose lungs had been mechanically ventilated for more than 72 hours and who died in our Respiratory Intensive Care Unit were included in the study. Patients with immunosuppression (organ transplantation, HIV infection and AIDS, severe neutropenia $<1.0 \times 10^{9} / 1$, steroid therapy equivalent to prednisone in a dose of $>20 \mathrm{mg} /$ day) were excluded. All patients received treatment with antibiotics during their stay in the ICU. Eight subjects $(32 \%)$ did not receive antibiotics during the 48 hours before death and 17 (68\%) received antibiotics until the day of death. The mean duration of antibiotic treatment was 9.5 (7.9) days. General characteristics of the study population and causes of ICU admission and death are presented in table 1.

The study was approved by the local ethical committee and family members gave written informed consent for the study to be performed.

CLINICAL AND RADIOGRAPHIC EVALUATION

All clinical and laboratory data were recorded on the day of death. The chest radiographs were also reviewed by one of the investigators and an independent radiologist on the day of death.

\section{SAMPLING METHODS}

Non-invasive and invasive sampling was performed 0-90 minutes after death without discontinuing mechanical ventilation through-

Table 1 Mean (SD) general characteristics of the study population

\begin{tabular}{|c|c|c|}
\hline & $\begin{array}{l}\text { Without } \\
\text { antibiotics } \\
(n=8)\end{array}$ & $\begin{array}{l}\text { With } \\
\text { antibiotics } \\
(n=17)\end{array}$ \\
\hline Age (years) & $52(1)$ & $57(17)$ \\
\hline $\mathrm{M} / \mathrm{F}$ & $22 / 6$ & $14 / 3$ \\
\hline APACHE II ${ }^{\star}$ & $20(2)$ & $21(7)$ \\
\hline $\begin{array}{l}\text { Duration of mechanical } \\
\text { ventilation (days) }\end{array}$ & $10(70)$ & $13(13)$ \\
\hline $\begin{array}{l}\mathrm{PaO}_{2} / \mathrm{FIO}_{2} \\
\text { Cause of ICU admission }\end{array}$ & $210(136)$ & $207(126)$ \\
\hline Acute respiratory failure & 5 & 7 \\
\hline Stroke & 1 & 5 \\
\hline Postoperative & 1 & 2 \\
\hline Cranial trauma & - & 3 \\
\hline Myocardial infarction & 1 & - \\
\hline Cause of death & & \\
\hline Hypoxaemia & 3 & 2 \\
\hline Multiple organ failure & 2 & 6 \\
\hline Cerebral death & 3 & 9 \\
\hline
\end{tabular}

NS for all quantitative comparisons

${ }^{\star}$ Acute physiology and chronic health evaluation determined on the day of death. out the procedures $\left(\mathrm{FIO}_{2}\right.$ set at 1.0). A tracheobronchial aspirate (TBA) was obtained (Moc Strap, La Llagosta, Spain) and bilateral fibreoptic bronchoscopic examinations were then performed using different bronchoscopes for each lung. A protected specimen brush (PSB) sample (Microbiology Brush, Mill-Rose Laboratories Inc, Mentor, Ohio, USA) was retrieved from the area of maximal inflammation corresponding to the area of chest radiographic infiltrates. Thereafter, protected bronchoalveolar lavage (BAL) fluid samples (Mill-Rose Laboratories Inc) were obtained by instilling an aliquot of $20 \mathrm{ml}$ sterile saline which was discarded, followed by four aliquots each of $30 \mathrm{ml}$ sterile saline. The recovery of BAL fluid was 26 (16) $\mathrm{ml}$. Conventional bronchoalveolar lavage was then performed in the same subsegment by instilling three aliquots of $50 \mathrm{ml}$ sterile saline. The recovered BAL fluid was 70 (35) $\mathrm{ml}$.

Immediate post-mortem lung biopsy specimens were then obtained. Through a thoracotomy incision one guided biopsy specimen from each lung was obtained with the aid of the light of the bronchoscope. The bronchoscope was guided to the area of maximal inflammation corresponding to chest radiographic infiltrates. In the absence of infiltrates the bronchoscope was guided to the lower lobes. In addition, following strict aseptic techniques, blinded bilateral pulmonary biopsies (not guided by fibreoptic bronchoscope) were obtained through the same thoracic incisions. The thoracotomy incision was done at the fifth intercostal space from the midclavicular to the midaxillary lines.

Three fragments were obtained from both superior and inferior lobes from each lung and two from the middle lobe giving an overall number of 16 biopsy specimens per patient (two guided in addition to 14 blinded). The total number of biopsy specimens was 375 (in three patients only one lung was sampled). In 14 patients (217 samples) the samples were obtained within 30 minutes after death, in five within 60 minutes (62 samples), and in six within 90 minutes (96 samples). Of those 14 patients in whom samples were obtained within 30 minutes of death, eight were organ donors and the period between brain death and sample extraction was less than 12-24 hours. In one organ donor the specimen was taken 30 60 minutes after death.

The average size of the lung biopsy specimens was $2 \times 2 \times 2 \mathrm{~cm}$. They were always obtained from peripheral zones of the lung. Each fragment was halved lengthwise with one specimen examined histologically and the other submitted for quantitative microbiological culture. All lung tissue specimens were embedded in paraffin and stained with haematoxylin/eosin, periodic acid Schiff, MayGrünwald Giemsa, and Gram stains. The pathologists were blinded to the microbiological results and clinical information. Guided and blind lung biopsy specimens were not analysed separately. 
MICROBIOLOGICAL PROCESSING

TBA and PSB specimens and protected BAL fluid were processed as described elsewhere. ${ }^{4}$ Biopsy samples were homogenised. Serial dilutions $\left(10^{-1}, 10^{-2}, 10^{-3}\right)$ of each sample were prepared in sterile normal saline and $0.1 \mathrm{ml}$ of each dilution was inoculated into different culture media ( $5 \%$ sheep blood, chocolate, CDC agar, and saboraud dextrosa). The cultures were incubated at $37^{\circ} \mathrm{C}$ under aerobic and anaerobic conditions and in a $\mathrm{CO}_{2}$ enriched atmosphere. All microorganisms isolated were identified by standard laboratory methods. ${ }^{13}$ Non-pathogenic microorganisms (Neisseria spp, Corynebacterium spp, streptococci other than $S$ pneumoniae and Candida albicans) were excluded from the analysis.

\section{DEFINITIONS}

Histological pneumonia: accumulation of polymorphonuclear leucocytes in the capillaries and adjacent alveolar spaces in at least one sample. ${ }^{14}$

Microbiologically active pneumonia: presence of both histological pneumonia and positive lung tissue cultures (as defined by a bacterial burden of $\geqslant 10^{3} \mathrm{cfu} / \mathrm{g}$ lung tissue) in the corresponding halves of at least one sample.

Clinical suspicion of pneumonia according to the criteria of Fohanson et al ${ }^{11}$ : clinical suspicion of pneumonia with an infiltrate on the chest radiograph and at least one of the following clinical criteria: leucocytosis $>12 \times 10^{9} / \mathrm{ml}$, fever $>38.3^{\circ} \mathrm{C}$, or the presence of purulent tracheobronchial secretions.

Clinical pulmonary infection score $(C P I S)^{12}$ : clinical suspicion of pneumonia in the presence of a positive score at different cut offs (0-12 points) which include the following variables: (1) body temperature; (2) blood leucocyte count and number of band forms; (3) character of tracheobronchial secretions (purulent or not) and quantity of tracheobronchial aspirates; (4) microscopic examination (Gram stain) and semiquantitative culture of bronchial secretions; (5) arterial oxygen tension/ inspiratory fraction of oxygen $\left(\mathrm{PaO}_{2} / \mathrm{FIO}_{2}\right)$; and (6) chest radiograph.

Diffuse alveolar damage: presence of interstitial or alveolar oedema, fibrin deposition, and initial development of hyaline membranes; or the presence of fibroblast proliferation within the interstitium and alveolar lining cell hyperplasia. ${ }^{15} 16$

Cut off points for sampling techniques: TBA specimens were considered positive in the presence of $\geqslant 10^{5} \mathrm{cfu} / \mathrm{ml}$, PSB specimens $\geqslant 10^{3} \mathrm{cfu} / \mathrm{ml}$, and protected $\mathrm{BAL}$ and $\mathrm{BAL}$ specimens $\geqslant 10^{4} \mathrm{cfu} / \mathrm{ml}$.

\section{STATISTICAL ANALYSIS}

Results are expressed as mean (SD). The Student's $t$ test was used to compare means. The accuracy of clinical diagnostic criteria for pneumonia was calculated using microbiologically active pneumonia as a reference test. Operative characteristics were calculated according to standard methods. The level of significance was set at $5 \%$.

\section{Results}

HISTOLOGY

The incidence of histological pneumonia in at least one sample was $92 \%$ (23 of 25 patients). Two subjects had no histological evidence of pneumonia, three had unilateral pneumonia, 17 others $(68 \%)$ had bilateral pneumonia and the remaining three patients, in whom the study was restricted to one lung, also had pneumonia.

Other additional histological diagnoses in patients with pulmonary infiltrates were: alveolar haemorrhage $(n=2)$, diffuse alveolar damage $(\mathrm{DAD})(\mathrm{n}=7)$, interstitial fibrosis $(\mathrm{n}=2)$, DAD plus interstitial fibrosis $(n=3)$, aspiration pneumonitis $(\mathrm{n}=1)$, pulmonary hypertension $(n=2)$, bronchiolitis obliterans $(n=3)$, and pulmonary thromboembolism $(\mathrm{n}=1)$. DAD always coexisted with histological pneumonia.

MICROBIOLOGY

Cultures of lung tissue revealed growth of 23 pathogenic microorganisms above the threshold in 14 patients (56\%). These included Gram positive pathogens in seven (Streptococcus pneumoniae $(\mathrm{n}=1)$, Staphylococcus aureus $(\mathrm{n}=2)$, methicillin-resistant Staphylococcus aureus (MRSA) $(\mathrm{n}=4))$, Gram negative pathogens in 15 (Proteus mirabilis $(\mathrm{n}=1)$, Serratia marcescens $(\mathrm{n}=1)$, Pseudomonas aeruginosa $(\mathrm{n}=9)$, Stenotrophomonas maltophilia $(\mathrm{n}=3)$, Acinetobacter anitratus $(\mathrm{n}=1)$ ), and Candida krusei in one (the latter also confirmed histologically).

\section{MICROBIOLOGICALLY ACTIVE PNEUMONIA}

The number of patients with both histological pneumonia and positive lung tissue cultures in the corresponding halves of at least one sample (and therefore conforming to the definition of microbiologically active pneumonia used as a reference test in the study) was $13(52 \%)$.

DIAGNOSTIC ACCURACY OF CLINICAL

PARAMETERS

Chest radiography

A total of 47 lungs were evaluated for radiographic infiltrates (three lungs could not be sampled due to technical problems during the procedure). Considering both lungs together, 13 patients (52\%) had localised and seven $(28 \%)$ had diffuse infiltrates. Sensitivity was high $(92 \%)$ but specificity was low $(33 \%)$. The diagnostic accuracy of infiltrates of the left lung was considerably higher than that of the right lung (table 2).

\section{Leucocytosis, fever, purulent secretions}

Leucocytosis was present in 15 patients $(60 \%)$, fever in $13(52 \%)$, and purulent secretions in $16(64 \%)$. The diagnostic accuracy of each of these three parameters was generally poor, the sensitivities ranging between $46 \%$ and $77 \%$ and the specificities between $42 \%$ and $58 \%$.

\section{Clinical criteria according to fohanson et al}

Two out of three clinical criteria in addition to the presence of infiltrates on the chest radiograph improved the diagnosis, with specificity increasing to $75 \%$ and sensitivity to $69 \%$. Sensitivity was not superior in the 
Table 2 Accuracy of clinical and radiographic variables as defined by fohanson et al ${ }^{11}$ for the diagnosis of VAP

\begin{tabular}{|c|c|c|c|c|}
\hline Variable or combination of variables & $\begin{array}{l}\text { Sensitivity } \\
\%(n)\end{array}$ & $\begin{array}{l}\text { Specificity } \\
\%(n)\end{array}$ & $\begin{array}{l}\text { Positive } \\
\text { predictive } \\
\text { value } \% \text { (n) }\end{array}$ & $\begin{array}{l}\text { Negative } \\
\text { predictive } \\
\text { value } \%(n)\end{array}$ \\
\hline Chest radiograph (right or left) & $92(12 / 13)$ & $33(4 / 12)$ & $60(12 / 20)$ & $80(4 / 5)$ \\
\hline Chest radiograph (right) & $73(8 / 11)$ & $33(4 / 12)$ & $50(8 / 16)$ & $57(4 / 7)$ \\
\hline Chest radiograph (left) & $92(11 / 12)$ & $75(9 / 12)$ & $79(11 / 14)$ & $90(9 / 10)$ \\
\hline Leucocytosis & $77(10 / 13)$ & $58(7 / 12)$ & $67(10 / 15)$ & $70(7 / 10)$ \\
\hline Fever & $46(6 / 13)$ & $42(5 / 12)$ & $46(6 / 13)$ & $42(5 / 12)$ \\
\hline Purulent secretions & $69(9 / 13)$ & $42(5 / 12)$ & $56(9 / 16)$ & $56(5 / 9)$ \\
\hline Chest radiograph + one of three criteria & $85(11 / 13)$ & $33(4 / 12)$ & $58(11 / 19)$ & $67(4 / 6)$ \\
\hline Chest radiograph + two of three criteria & $69(9 / 13)$ & $75(9 / 12)$ & $75(9 / 12)$ & $69(9 / 13)$ \\
\hline Chest radiograph + all three criteria & $23(3 / 13)$ & $92(11 / 12)$ & $75(3 / 4)$ & $52(11 / 21)$ \\
\hline
\end{tabular}

Sensitivity $=$ rate of correctly classified patients with VAP and total number of patients with VAP, specificity $=$ rate of correctly classified patients without VAP and total number of patients withou VAP; positive predictive value $=$ rate of correctly classified patients with VAP and total number of patients classified as having VAP (correctly positive plus false positive); negative predictive value = rate of correctly classified patients without VAP and total number of patients classified as not having VAP (correctly negative plus false negative).

Table 3 Accuracy of non-invasive and invasive diagnostic techniques for ventilator associated pneumonia

\begin{tabular}{llccc}
\hline & $\begin{array}{l}\text { Sensitivity } \\
\%(n)\end{array}$ & $\begin{array}{l}\text { Specificity } \\
\%(n)\end{array}$ & $\begin{array}{l}\text { Positive } \\
\text { predictive } \\
\text { value \% }(n)\end{array}$ & $\begin{array}{l}\text { Negative } \\
\text { predictive } \\
\text { value \% (n) }\end{array}$ \\
\hline TBiagnostic technique & $69(9 / 13)$ & $92(11 / 12)$ & $90(9 / 10)$ & $73(11 / 15)$ \\
Protected BAL & $39(5 / 13)$ & $100(12 / 12)$ & $100(5 / 5)$ & $60(12 / 20)$ \\
BAL & $77(10 / 13)$ & $58(7 / 12)$ & $67(10 / 15)$ & $70(7 / 10)$ \\
PSB & $62(8 / 13)$ & $75(9 / 12)$ & $73(8 / 11)$ & $64(9 / 14)$ \\
$\begin{array}{l}\text { Any invasive diagnostic technique } \\
\begin{array}{l}\text { Any non-invasive or invasive } \\
\text { diagnostic technique }\end{array}\end{array}$ & $85(11 / 13)$ & $50(6 / 12)$ & $65(11 / 17)$ & $75(6 / 8)$ \\
\hline
\end{tabular}

$\mathrm{TBA}=$ tracheobronchial aspirates $; \mathrm{BAL}=$ bronchoalveolar lavage $\mathrm{PSB}=$ protected specimen brush.

For definition of terms see footnote to table 2 .

Table 4 Accuracy of clinical criteria and non-invasive and invasive diagnostic techniques for ventilator associated pneumonia stratified according to whether or not antibiotics were given during the last 48 hours before death

\begin{tabular}{|c|c|c|c|c|}
\hline \multirow[b]{2}{*}{ Diagnostic method } & \multicolumn{2}{|c|}{ Without antibiotics $(n=8)$} & \multicolumn{2}{|c|}{ With antibiotics $(n=17)$} \\
\hline & $\begin{array}{l}\text { Sensitivity } \\
\%(n)\end{array}$ & $\begin{array}{l}\text { Specificity } \\
\%(n)\end{array}$ & $\begin{array}{l}\text { Sensitivity } \\
\%(n)\end{array}$ & $\begin{array}{l}\text { Specificity } \\
\%(n)\end{array}$ \\
\hline${\text { Clinical }{ }^{\star}}^{*}$ & $67(4 / 6)$ & $0(0 / 2)$ & $71(5 / 7)$ & $90(9 / 10)$ \\
\hline TBA & $83(5 / 6)$ & $100(2 / 2)$ & $57(4 / 7)$ & $90(9 / 10)$ \\
\hline Protected BAL & $17(1 / 6)$ & $100(2 / 2)$ & $57(4 / 7)$ & $100(10 / 10)$ \\
\hline BAL & $67(4 / 6)$ & $50(1 / 2)$ & $86(6 / 7)$ & $60(6 / 10)$ \\
\hline PSB & $83(5 / 6)$ & $100(2 / 2)$ & $43(3 / 7)$ & $70(7 / 10)$ \\
\hline Any invasive & $83(5 / 6)$ & $50(1 / 2)$ & $86(6 / 7)$ & $50(5 / 10)$ \\
\hline Any non-invasive or invasive & $83(5 / 6)$ & $50(1 / 2)$ & $86(6 / 7)$ & $50(5 / 10)$ \\
\hline
\end{tabular}

${ }^{\star}$ Chest radiograph infiltrates plus two of the following: leucocytosis, purulent secretions, fever.

$\mathrm{TBA}=$ tracheobronchial aspirates; $\mathrm{BAL}=$ bronchoalveolar lavage $; \mathrm{PSB}=$ protected specimen brush.

Table 5 Association of clinical and microbiological diagnoses for ventilator associated pneumonia

\begin{tabular}{|c|c|c|c|c|}
\hline $\begin{array}{l}\text { Histology + lung } \\
\text { tissue culture }\end{array}$ & $\begin{array}{l}\text { Clinical diagnosis } \\
\text { (chest radiograph }+ \\
\text { two of three clinical } \\
\text { criteria) }\end{array}$ & $\begin{array}{l}\text { Non-invasive } \\
\text { microbiological } \\
\text { diagnosis (TBA) }\end{array}$ & $\begin{array}{l}\text { Invasive } \\
\text { microbiological } \\
\text { diagnosis (PSB, } \\
B A L, \text { protected } \\
B A L)\end{array}$ & $\begin{array}{l}\text { Both } \\
\text { non-invasive } \\
\text { and invasive } \\
\text { microbiological } \\
\text { diagnoses }\end{array}$ \\
\hline Positive & False negative & Adequate & Adequate & Adequate \\
\hline (Pneumonia present) & $(\mathrm{n}=4)$ & $3 / 4(75 \%)$ & $3 / 4(75 \%)$ & $3 / 4(75 \%)$ \\
\hline Negative & False positive & Adequate & Adequate & Adequate \\
\hline (Pneumonia absent) & $(\mathrm{n}=3)$ & $3 / 3(100 \%)$ & $1 / 3(33 \%)$ & $2 / 3(67 \%)$ \\
\hline Positive & Correct positive & Inadequate & Inadequate & Inadequate \\
\hline (Pneumonia present) & $(\mathrm{n}=9)$ & $3 / 9(33 \%)$ & $1 / 9(11 \%)$ & $1 / 9(11 \%)$ \\
\hline Negative & Correct negative & Inadequate & Inadequate & Inadequate \\
\hline (Pneumonia absent) & $(\mathrm{n}=9)$ & $1 / 9(11 \%)$ & $4 / 9(44 \%)$ & $5 / 9(55 \%)$ \\
\hline
\end{tabular}

Diagnostic techniques (non-invasive, invasive, or both) are stratified according to the impact which its results would have had on clinical decisions.

Adequate $=$ antibiotic treatment would have been administered or withheld adequately in the presence of a false negative or positive clinical diagnosis, respectively; inadequate $=$ antibiotic treatment would have been administered or withheld inadequately in the presence of a correct positive or negative clinical diagnosis, respectively.

$\mathrm{TBA}=$ tracheobronchial aspirates; $\mathrm{BAL}=$ bronchoalveolar lavage; $\mathrm{PSB}=$ protected specimen brush. absence of antibiotic treatment $(67 \%)$. The presence of all three criteria increased the specificity to $92 \%$ at the cost of a high beta error (sensitivity $23 \%$ ).

\section{Clinical pulmonary infection score (CPIS)}

The use of a Pugin score of $>6$ as recommended by Pugin et $a l^{12}$ resulted in a sensitivity of $77 \%$ and a specificity of $42 \%$.

DIAGNOSTIC ACCURACY OF NON-INVASIVE AND

INVASIVE SAMPLING TECHNIQUES

The accuracies of TBA, PSB, BAL fluid, and protected BAL fluid specimens for the presence of pneumonia with microbiologically active pneumonia as the reference test are given in table 3. BAL fluid had the highest sensitivity $(77 \%)$ whereas protected BAL fluid had the highest specificity (100\%). TBA specimens had the highest diagnostic accuracy (20/25, $80 \%)$ and balanced sensitivity (69\%) and specificity $(92 \%)$. The combined use of all invasive diagnostic techniques (PSB, BAL fluid and protected BAL fluid) increased the sensitivity to $85 \%$ but at the cost of a beta error of $50 \%$. These figures were identical when TBA was added as a non-invasive tool.

Sensitivity and specificity did not change substantially when stratified according to antibiotic treatment. This was especially true when comparing the combined invasive as well as invasive and non-invasive techniques (table 4).

IMPACT OF MICROBIOLOGICAL DIAGNOSIS ON THE ACCURACY OF DIAGNOSTIC EVALUATION FOR THE PRESENCE OF PNEUMONIA

In four cases with a clinically false negative diagnosis non-invasive and invasive sampling methods would correctly have established pneumonia in three $(75 \%)$. Conversely, in three cases with a clinically false positive diagnosis TBA would have correctly identified the absence of pneumonia in all three and invasive sampling methods in one $(33 \%)$.

On the other hand, an inadequate diagnosis would have been established by TBA in three cases $(33 \%)$ of nine correctly diagnosed clinically as having pneumonia and invasive sampling methods in one $(11 \%)$, while in another nine cases correctly classified as not having pneumonia an inadequate diagnosis would have been provided by TBA in one case $(11 \%)$ and invasive sampling methods in four $(44 \%)$. These numbers would have been three $(75 \%)$ and two $(67 \%)$, and one $(11 \%)$ and five $(55 \%)$, respectively, if both non-invasive and invasive techniques were used together. Thus, adequate diagnoses from microbiological investigation were achieved in five cases and inadequate diagnoses in six (table 5).

\section{Discussion}

This study offers the following three important findings: (1) the accuracy of clinical criteria (infiltrates on the chest radiograph and two of the following: leucocytosis, fever, purulent secretions) for the diagnosis of pneumonia was reasonable (sensitivity $69 \%$, specificity $75 \%$ ) and the CPIS was not more accurate; (2) non- 
invasive (TBA) and invasive (PSB, BAL, protected BAL) sampling techniques were not superior to these clinical criteria; and (3) when microbiological results were added to clinical criteria, adequate diagnoses originating from the microbiological investigation which might have corrected false positive and false negative clinical judgements were countered by a similar proportion of inadequate diagnoses.

Three crucial methodological issues must be borne in mind before interpreting these results. Firstly, it must be recognised that there is no irrefutable reference test for the validation of diagnostic criteria and techniques. Specific limitations of the post-mortem lung biopsy approach include the selection of a population not necessarily representative of all mechanically ventilated patients, the risk of an overestimate of lung injuries in histological samples inherent in the study of severely ill patients, and sampling errors due to the multifocal nature of VAP. To minimise these potential pitfalls we used a combination of the histological presence of pneumonia with positive quantitative cultures of lung tissue samples as the reference test. We thought that this approach would allow us to distinguish between foci of residual pneumonia and active and clinically symptomatic pneumonia. It would also represent a rigorous criterion to protect against confounding colonisation with pneumonia.

Secondly, the clinical parameters were calculated on the day of death, a fact that does not necessarily correspond with the evolution of pneumonia, creating a timing discrepancy between both events. The criterion of a new infiltrate on the chest radiograph originally required by Johanson et al ${ }^{11}$ could not be met in the majority of patients because of the study design. It should therefore be recognised that our approach does not exactly apply to the usual clinical situation in which a patient with suspected VAP is evaluated. It represents an evaluation of diagnostic criteria and techniques in the prevalence of microbiologically active pneumonia on the day of death. Although we ignore the exact extent to which these results can be extrapolated to the regular clinical situation, we would argue that our results provide a reasonable approximation to it.

Finally, we defined one single (guided or blinded) histologically and microbiologically positive sample as indicating the presence of VAP. The definition was based on the recognition of the generally multifocal spread of VAP. ${ }^{126-9}{ }^{24}$ This approach is in contrast to other studies where the diagnostic accuracy of different sampling techniques for the presence of pneumonia in lung tissue samples was assessed. ${ }^{49}$ Although the latter approach offers important insights into the diagnostic value of different sampling techniques compared with each other, it is not an accurate means of demonstrating its clinical value. Instead, the only clinically meaningful analysis is to determine the ability of clinical criteria and different sampling techniques to diagnose the presence of VAP in the individual patient.

We found that the presence of chest radiographic infiltrates was the most sensitive clinical parameter for diagnosing VAP. However, the rate of false positive results was as high as $67 \%$. Few other studies have investigated the diagnostic value of portable chest radiography for VAP. Only three studies ${ }^{145}$ have compared chest radiographs with the histological findings of pneumonia. The sensitivities reported from these studies were $57 \%$, $78 \%$, and $87 \%$ while the specificities were $70 \%, 42 \%$, and $58 \%$, respectively. These differences are largely explained by the fact that lung tissue from the radiographically involved area was used as a reference rather than from remote areas of the lung. Nevertheless, two of these three studies confirmed the general tendency to a higher sensitivity than specificity. The high rate of false positive results is probably due to alternative diagnoses that may cause pulmonary infiltrates mimicking VAP such as alveolar haemorrhage, atelectasis, pulmonary infarction, and the fibroproliferative phase of ARDS. ${ }^{17}$ False negative results may result from the initial phases of pneumonia not detected on the plain chest radiograph. In a previous study we found that the initial phases of pneumonia represented $11 \%$ of the total histological patterns of pneumonia. ${ }^{14}$ The technical limitations of portable chest radiography may hinder interpretation of radiographs. ${ }^{18}$ Interestingly, the diagnostic accuracy of the left chest radiograph was considerably higher than that of the right. A possible explanation for this may be the higher incidence of atelectasis on the right side.

Leucocytosis and purulent secretions had a remarkable sensitivity but only poor specificity. Few authors have addressed leucocytosis alone as a clinical parameter for diagnosing VAP and compared it with histological examination. Andrews et al found a sensitivity of $100 \%$ and a specificity of $20 \%$ in patients with ARDS. ${ }^{1}$ Obviously, leucocytosis may be induced by a wide variety of clinical conditions. The high rate of false positive results of purulent secretions is easily explained by the fact that almost all mechanically ventilated patients have purulent bronchitis, particularly during prolonged periods. Rouby et $a l^{19}$ found that bronchiolitis without pneumonia was not an infrequent finding in mechanically ventilated patients. In contrast, false negative results may be due to foci of pneumonia located at the periphery which impair the passage of these secretions to the more central airways. In addition, the presence and degree of purulence and even the volume of tracheobronchial secretions are subjective parameters which may cause a bias.

The presence of fever was not a valid predictor of VAP. Andrews and colleagues ${ }^{1}$ found that fever had $100 \%$ sensitivity and $20 \%$ specificity for diagnosing pneumonia in patients with ARDS. In a previous study ${ }^{4}$ we also found that this parameter had a sensitivity of $55 \%$ and a specificity of $58 \%$. These results are not surprising since a certain degree of immunosuppression is common in critically ill patients, impairing the body temperature response to infections. The prevalence of fever may be explained by mechanisms other than pneumo- 
nia. Meduri and coworkers ${ }^{20}$ found that only $42 \%$ of patients with fever and infiltrates on the chest radiograph had pneumonia; most had fever due to infectious foci other than pneumonia. They also suggested that the fibroproliferative phase of ARDS may frequently occur with fever, thus mimicking pneumonia. ${ }^{17}$

The combination of infiltrates on the chest radiograph with two of three clinical criteria had a reasonable diagnostic accuracy. Similarly, clinical criteria yielded a sensitivity of $62 \%$ and a specificity of $84 \%$ in a study using microbiological criteria for VAP. ${ }^{3}$ Two necropsy studies have also shown similar results (sensitivity $60 \%$ and specificity $80 \%$ ) when evaluating clinical criteria. ${ }^{12}$ Conversely, a previous study from our group ${ }^{4}$ and a more recent one by Kirtland et $a l^{10}$ found that clinical criteria predicted the presence of histological pneumonia only poorly. In our view these results reflect limitations inherent in reference tests based on a single biopsy specimen and histological examination, without confirmation of microbiological activity.

The CPIS was not found to be superior to the clinical criteria recommended by Johanson et al. ${ }^{11}$ It achieved a sensitivity of $77 \%$ but the rate of false positives was $58 \%$. Factors responsible for the high rate of false positive results may have been the number of leucocyte band forms, the quantity of tracheobronchial secretions, Gram stains, and the $\mathrm{PaO}_{2} / \mathrm{FIO}_{2}$. The modified CPIS score has been clinically validated by Garrard et $a l^{11}$ in a unique population who had not received any previous antibiotic treatment. They found that serial determinations of the CPIS followed a similar pattern to quantitative cultures of nondirected bronchoalveolar lavage in both patients who responded to antibiotics and those who did not. In a post-mortem study using combined histology and lung microbiology as the reference test, Papazian and colleagues found a sensitivity of $72 \%$ and a specificity of $85 \%$ for the CPIS. ${ }^{6}$ These results do not indicate a superiority of the CPIS to Johanson's criteria and, given that the CPIS is more time consuming to calculate, there is no evidence to recommend this score in routine clinical practice.

The diagnostic accuracy of non-invasive and invasive sampling techniques was comparable to that found in previous post-mortem lung biopsy studies. ${ }^{46-10}$ The application of all invasive (or all invasive and non-invasive) techniques together increased the sensitivity but also the beta error. They were not superior to the diagnostic accuracy of clinical criteria. This was true irrespective of previous antibiotic treatment. However, from a clinical point of view a more important consideration is the impact of the microbiological data on correcting false positive results from clinical judgement, thereby avoiding overuse of antibiotics, and the cost of introducing new false negative results. In this regard, sampling techniques proved to be of limited value since TBA, but not invasive techniques, correctly diagnosed the absence of pneumonia in all cases. On the other hand, in those patients correctly diagnosed as having pneumonia by clinical criteria, non-invasive and invasive sampling techniques failed to yield positive results in some cases. These patients would not have received antibiotics and, as a consequence, would have been at high risk of death due to misdiagnosis. Conversely, if patients were screened for infection without clinical suspicion, a considerable number of patients would have been adequately treated at the cost of a similar number of patients with false positive microbiological results who would have been overtreated. Thus, our results suggest that it may be hazardous to base decisions on antibiotic treatment exclusively on the results of sampling techniques. The risk of death in patients with VAP not receiving antibiotic treatment is probably higher than the corresponding risk of overuse of antibiotics. Negative microbiological results in the presence of clinically suspected VAP must not therefore be a reason for withholding antibiotics unless an alternative diagnosis is clearly established.

In summary, our results confirm that clinical parameters are reasonably accurate in the diagnosis of VAP and are still the principal criteria in establishing a diagnosis. We believe that the key point in clinical practice is to find a balance between the information provided by clinical judgement and the microbiology of the lower airways, and not to withhold antibiotics if VAP is clinically suspected.

Dr Santiago Ewig was a 1998 research fellow from the Medizinische Universitätsklinik and Poliklinik Bonn, Bonn, Germany; Dr Torsten Bauer was a 1998 research fellow from the Berufsgenossenschaftliche Kliniken Bergmannsheil-UniversitätsBerufsgenossenschaftliche Kliniken Bergmannsheil-Universitätsklinik, Ruhr-Universitätsklinik Bochum, Bochum, Germany; and Dr Hernan Cabellos was a 1996 research fellow from the Hospital Clínico de la Universidad de Chile, Santiago de Chile, Chile. This study was supported in part by the following grants: Commisionat per a Universitats i Recerca de la Generalitat de Catalunya 1997 SGR 00086, Fundació Clínic/CIRIT, IDIBAPS Hospital Clínic Barcelona, FIS (Fondo de Investigación Sanitaria) $94 / 0583$, and I+D 96/0024

1 Andrews CP, Coalson JJ, Smith JD, et al. Diagnosis of nosocomial bacterial pneumonia in acute diffuse lung injury. comial bacterial pneum

2 Bell RC, Coalson JJ, Smith JD, et al. Multiple organ system failure and infection in adult respiratory distress syndrome. Ann Intern Med 1983;99:293-8.

3 Fagon JY, Chastre J, Hance AJ, et al. Evaluation of clinical judgement in the identification and treatment of nosocomial pneumonia in ventilated patients. Chest 1993;103 547-53.

4 Torres A, El-Ebiary M, Padró L, et al. Validation of different techniques for the diagnosis of ventilator-associated pneumonia. Am $\mathcal{F}$ Respir Crit Care Med 1994;149:324-31.

5 Wunderink RG, Woldenberg LS, Zeiss J, et al. The radiologic diagnosis of autopsy-proven ventilatorradiologic diagnosis Chest 1992;101:458-63.

6 Papazian L, Thomas P, Garbe L, et al. Bronchoscopic or blind sampling techniques for the diagnosis of ventilatorblind sampling techniques for the diagnosis of ventilator1995;152:1982-91.

7 Chastre J, Fagon JY. Invasive diagnostic testing should be routinely used to manage ventilated patients with suspected pneumonia. Am $f$ Respir Crit Care Med 1994;150:570-4

8 Marquette $\mathrm{CH}$, Copin MC, Wallet F, et al. Diagnostic tests for pneumonia in ventilated patients: prospective evaluation of diagnostic accuracy using histology as a diagnostic gold standard. Am 7 Respir Crit Care Med 1995;151: 1878-8.

9 Chastre J, Fagon JY, Bornet-Lesco M, et al. Evaluation of bronchoscopic techniques for the diagnosis of nosocomial pneumonia. Am 7 Respir Crit Care Med 1995;152. 231-40.

10 Kirtland SH, Corley DE, Winterbauer RH, et al. The diagnosis of ventilator-associated pneumonia. A comparison of histologic, microbiologic, and clinical criteria. Chest 1997;112:445-57. 
11 Johanson WG, Pierce Ak, Sandford JP, et al. Nosocomial respiratory infections with gram-negative bacilli: the significance of colonization of the respiratory tract. Ann Intern Med 1972;77:701-6.

12 Pugin J, Auckenthaler R, Mili N, et al. Diagnosis of ventilator-associated pneumonia by bacteriologic analysis of bronchoscopic and nonbronchoscopic "blind" bronchoalveolar lavage fluid. Am Rev Respir Dis 1991;143:1121-9.

13 Balows A, Harsler WJ, Jr. Manual of clinical microbiology, 5th edn, Section III. Washington, DC: American Society for Microbiology, 1991: 209-553.

14 Fàbregas N, Torres A, El-Ebiary M, et al. Histopathological and microbiological aspects of ventilator-associated pneumonia. Anesthesiology 1996;84;760-71.

15 Katzenstein AL, Askin FB. Diffuse alveolar damage, acute interstitial pneumonia, bronchiolitis obliterans-organizing pneumonia. In: Katzenstein AA, Askin FB, Livolsi VA, et al, pneumonia. In: Katzenstein AA, Askin FB, Livolsi VA, et al, neoplastic lung disease, 3rd edition. Philadelphia: WB Saunders, 1997:14-47.
16 Murray JF, Matthay MA, Luce JM, et al. An expanded definition of the adult respiratory distress syndrome. Am Rev Respir Dis 1988;138:720-3.

17 Meduri GU, Belenchia JM, Estes RJ, et al. Fibroproliferative phase of ARDS. Clinical findings and effects of corticosteroids. Chest 1991;100:943-52.

18 Lefcoe MS, Fox GA, Leasa DJ, et al. Accuracy of portable chest radiography in the critical care setting. Diagnosis of pneumonia based on quantitative cultures obtained from protected brush catheter. Chest 1994;105:885-7.

19 Rouby JJ, Martin de Lassale EM, Poete P, et al. Nosocomia bronchopneumonia in the critically ill. Am Rev Respir Dis 1992;146:1059-66.

20 Meduri GU, Mauldin GL, Wunderink RG, et al. Causes of fever and pulmonary densities in patients with clinical manifestations of ventilator-associated pneumonia. Chest 1994;106:221-35.

21 Garrard CS, A'Court CD. The diagnosis of pneumonia in the critically ill. Chest 1995;108:17-25S. 